\title{
Comment on Zhou et al. entitled "High plasma D-dimer level is associated with decreased survival in patients with lung cancer: a meta-analysis based on current evidence"
}

\author{
Shu Zhang • Ping Zhan • Yong Song
}

Received: 1 August 2013 / Accepted: 11 September 2013 / Published online: 26 September 2013

(C) International Society of Oncology and BioMarkers (ISOBM) 2013

To the Editor,

I read with great interest the article by Zhou et al. [1] evaluating the impact of high level of plasma D-dimer on the prognosis of lung cancer by conducting a metaanalysis, with the end point being survival time. Nevertheless, some methodological issues need to be addressed concerning the data provided in the meta-analysis by Zhou et al. [1].

1. Five electronic databases (PubMed, Embase, Chinese Biomedical Literature Database, Wanfang Database, and China National Knowledge Infrastructure (CNKI)) for studies were systematically searched by the authors. However, the authors had not focused specifically or in any details on the issue of the completeness of the search strategy report for databases. Search strategy report plays an important role in systematic reviews.

2. With respect to the assessment of study quality, two investigators independently evaluated the quality of each study. However, we would like to know how to solve the problems if there are discrepancies between the two authors. Besides, the authors used the Cochrane Handbook 5.0 Quality evaluation criteria to evaluate methodological quality of included studies. However, there were no detailed scores for each trial.
3. The $P$ value of Cochran's $Q$ statistic was used to investigate the degree of heterogeneity between the studies in the meta-analysis. The authors suggested that a $P$ value of more than the nominal level of 0.05 for the $Q$ statistic indicated a lack of heterogeneity across studies, and a fixed effects model should be used to pool the data. However, because of the low power of Cochran's $Q$ statistic, it was widely accepted that the heterogeneity was considered significant when the $P$ value of Cochran's $Q$ statistic is $<0.10$, while allowing the use of random effects model to pool the results.

4. In part of the results, the funnel plot was used to estimate publication bias, and the symmetric inverse funnel distribution was obtained. However, it is not appropriate to assess the publication bias for adjusted OR, as the number of studies was only 7 . As we know, a funnel plot should be inspected visually to assess for publication bias, in meta-analyses with a sufficient number of studies $(>9)$.

Moreover, more carefully and scientifically designed studies with large samples are still needed. We believe that these remarks will further contribute for more accurate elaboration and substantiation of the original results presented by Zhou et al. [1].

\section{Reference}

1. Zhou YX, Yang ZM, Feng J, et al. High plasma D-dimer level is associated with decreased survival in patients with lung cancer: a metaanalysis. Tumour Biol. 2013. doi:10.1007/s13277-013-0953-2. 